\title{
An improved Morgan-voyce collocation method for numerical solution of multi-pantograph equations
}

\author{
Ozgul Ilhan
}

Department of Mathematics, Faculty of Science, Mugla Sitki Kocman University, Mugla, Turkey

Received: 26 May 2017, Accepted: 14 June 2017

Published online: 25 December 2017.

\begin{abstract}
In this article, an improved collocation method based on the Morgan-Voyce polynomials for the approximates solution of multi-pantograph equations is introduced. The method is based upon the improvement of Morgan-Voyce polynomial solutions with the aid of the residual error function. First, the Morgan-Voyce collocation method is applied to the multi-pantograph equations and then Morgan-Voyce polynomial solutions are obtained. Second, an error problem is constructed by means of the residual error function and this error problem is solved by using the Morgan-Voyce collocation method. By summing the Morgan-Voyce polynomial solutions of the original problem and the error problem, we have the improved Morgan-Voyce polynomial solutions. When the exact solution of problem is not known, the absolute error can then be approximately computed by the Morgan-Voyce polynomial solution of the error problem. Numerical examples that the pertinent features of the method are presented. We have applied all of the numerical computations on computer using a program written in MATLAB.
\end{abstract}

Keywords: Morgan-Voyce polynomials, Pantograph equations, approximation methods, collocation points, residual error function.

\section{Introduction}

Functional-differential equations with proportional delays are usually refered to as pantograph equations or generalized equations. The name pantograph came from the work of Ockendon and Tayler [1] on the collection of current by the pantograph head of an electric locomotive.

We see these equations in many applications such as number theory, non-linear dynamical systems, electrodynamics, probality theory on algebraic structures, astrophysics, cell growth and quantum mechanics, among others. Several authors have studied proporties of the analytic solution of these equations and numerical methods. For example, the equations with variable coefficients are treated in [2-4].

In recent years, the numerical treatment of the pantograph equations of the retarded and advanced type has attracted attention. The existence of compactly supported solutions is a specific property for this type [5].

Pantograph equations are important for explanining many different phenomena. Specially, they turn out to be the fundamental equations when ODEs-based model fail. These equations are seen in studies based on biology, economy, control and electrodynamics [6,7] and in industrial equations [1,8].This phenomena has been studied in [8-10], and has direct applications to approximation theory and wavelets [10].

Since the beginning of 1994, to find the approximate solutions of differential, difference, integral and integro-differential-difference equations, multi-pantograph and generalized pantograph, Taylor, Chebyshev, Bessel and Legendre matrix methods have been used by Sezer et al [11-18]. 
The purpose of this work is to apply Morgan-Voyce polynomials to the nonhomogenous and the homogenous multi-pantograph equations with variable coefficients, which is the extension of the pantograph equations given in $[5,16]$.

In this study, we want to solve the pantograph equation given by

$$
y^{\prime}(t)=\lambda y(t)+\sum_{j=1}^{J} \mu_{j}(t) y\left(q_{j} t\right)+g(t), \quad 0 \leq t \leq b
$$

that is a multi-pantograph equations given in [5,19-21] and its initial condition is

$$
y(0)=\gamma
$$

In here, $y(t)$ is an unknown function, the known functions $\mu_{j}(t)$ and $g(t)$ are defined on interval $a \leq t \leq b$ and $\lambda$ and $q_{j}$ are real or complex constants.

In this paper, by improving the Morgan-Voyce collocation method with the aid of residual error function used in [24-26], we obtain an approximate solution of (1) expressed in the truncated Morgan-Voyce series form

$$
y_{N, M}(t)=y_{N}(t)+e_{N, M}(t)
$$

where

$$
y_{N}(t)=\sum_{n=0}^{N} a_{n} B_{n}(t)
$$

is the Morgan-Voyce solution and

$$
e_{N, M}(t)=\sum_{n=0}^{M} a_{n}^{*} B_{n}(t)
$$

is the Morgan-Voyce polynomial solution of the error problem obtained with the aid of the residual error function. Here $a_{n}, a_{n}^{*}, n=0,1,2, \ldots, N$ are the unknown Morgan-Voyce coefficients; $N$ and $M$ are any chosen positive integers such that $M \geq N \geq 2$; and $B_{n}(t), n=0,1,2, \ldots, N$ are the Morgan-Voyce polynomials defined by

$$
B_{n}(t)=\sum_{k=0}^{n}\left(\begin{array}{c}
n+k+1 \\
n-k
\end{array}\right) t^{k}, n \in \mathbb{N}
$$

\section{Fundamental matrix relations}

Firstly, we can write the Morgan-Voyce polynomials $B_{n}(t)$ in the matrix form as

$$
\mathbf{B}^{T}(t)=\mathbf{R T}^{T}(t) \Leftrightarrow \mathbf{B}(t)=\mathbf{T}(\mathbf{t}) \mathbf{R}^{T}
$$

where

$$
\mathbf{B}(\mathbf{t})=\left[B_{0}(t) B_{1}(t) \ldots B_{N}(t)\right], \mathbf{T}(t)=\left[\begin{array}{llll}
1 & t^{1} & t^{2} \ldots t^{N}
\end{array}\right]
$$

and

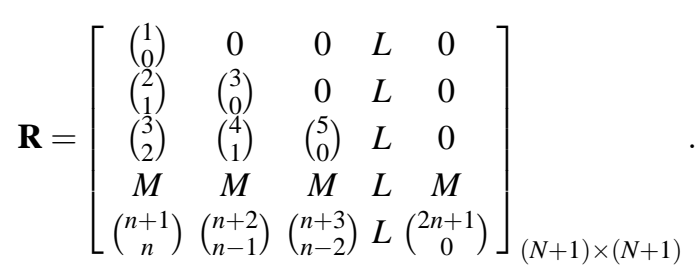


We consider the desired solution of Eq. (1) defined by the truncated Morgan-Voyce series (4). So we can write the finite series (4) in matrix form

$$
y(t)=\mathbf{B}(\mathbf{t}) \mathbf{A} ; \quad \mathbf{A}=\left[\begin{array}{llll}
a_{0} & a_{1} & \ldots & a_{N}
\end{array}\right]^{T}
$$

or from Eq. (5)

$$
y(t)=\mathbf{T}(\mathbf{t}) \mathbf{R}^{\mathbf{T}} \mathbf{A} .
$$

On the other hand, the relation between the matrix $\mathbf{T}(t)$ and its derivative is $\mathbf{T}^{(1)}(t)$ is

$$
\mathbf{T}^{(1)}(t)=\mathbf{T}(t) \mathbf{C}^{T}, \quad \mathbf{T}^{(0)}(t)=\mathbf{T}(t)
$$

where

$$
\mathbf{C}^{T}=\left[\begin{array}{ccccccc}
0 & 1 & 0 & L & 0 & 0 & 0 \\
0 & 0 & 2 & L & 0 & 0 & 0 \\
0 & 0 & 0 & L & 0 & 0 & 0 \\
M & M & M & M & M \\
0 & 0 & 0 & L & 0 & 0 & N \\
0 & 0 & 0 & L & 0 & 0 & 0
\end{array}\right]
$$

If we follow from (6) and (7), we derive

$$
y^{\prime}(t)=\mathbf{B}^{\prime}(t) \mathbf{A}=\mathbf{T}^{\prime}(t) \mathbf{R}^{T} \mathbf{A}=\mathbf{T}(t) \mathbf{C}^{T} \mathbf{R}^{T} \mathbf{A} .
$$

Similarly, the matrix relations are as follows,

$$
\begin{gathered}
\mathbf{T}\left(q_{j} t\right)=\mathbf{T}(t) \mathbf{B}\left(q_{j}\right) \\
y\left(q_{j} t\right)=\mathbf{T}(t) \mathbf{B}\left(q_{j}\right) \mathbf{R}^{T} \mathbf{A}
\end{gathered}
$$

where

$$
B\left(q_{j}\right)=\left[\begin{array}{cccc}
\left(q_{j}\right)^{0} & 0 & K & 0 \\
0 & \left(q_{j}\right)^{1} & K & 0 \\
M & M & 0 & M \\
0 & 0 & K & \left(q_{j}\right)^{N}
\end{array}\right]
$$

\section{Method of solution}

Now, we are ready to construct the fundamental matrix equation for Eq. (1). For this aim, by substituting the matrix relation (6),(8) and (9) into Eq. (1), we obtain the matrix equation

$$
\mathbf{T}(t) \mathbf{C}^{T} \mathbf{R}^{T} \mathbf{A}=\lambda \mathbf{T}(t) \mathbf{R}^{T} \mathbf{A}+\sum_{j=1}^{J} \mu_{j}(t) \mathbf{T}(t) \mathbf{B}\left(q_{j}\right) \mathbf{R}^{T} \mathbf{A}+\mathbf{g}(\mathbf{t})
$$

In Eq. (10) we substitute collocation points defined by

$$
t_{i}=\frac{b}{N} i, \quad i=0,1, \ldots, N
$$

and we obtain the system of the matrix equations as,

$$
\mathbf{T}\left(t_{i}\right) \mathbf{C}^{T} \mathbf{R}^{T} \mathbf{A}=\lambda \mathbf{T}\left(t_{i}\right) \mathbf{R}^{T} \mathbf{A}+\sum_{j=1}^{J} \mu_{j}\left(t_{i}\right) \mathbf{T}\left(t_{i}\right) \mathbf{B}\left(q_{j}\right) \mathbf{R}^{T} \mathbf{A}+\mathbf{g}\left(\mathbf{t}_{i}\right), \quad i=0,1, \ldots, N
$$


or briefly the fundamental matrix equation

$$
\left\{\mathbf{T C}^{T} \mathbf{R}^{T}-\lambda \mathbf{T} \mathbf{R}^{T}-\sum_{j=1}^{J} \mathbf{M}_{j} \mathbf{T B}\left(q_{j}\right) \mathbf{R}^{T}\right\} \mathbf{A}=\mathbf{G}
$$

where

$$
\mathbf{M}_{j}=\left[\begin{array}{cccc}
\mu_{j}\left(t_{0}\right) & 0 & L & 0 \\
0 & \mu_{j}\left(t_{1}\right) & L & 0 \\
M & M & 0 & 0 \\
0 & 0 & L & \mu_{j}\left(t_{N}\right)
\end{array}\right], G=\left[\begin{array}{c}
g\left(t_{0}\right) \\
g\left(t_{1}\right) \\
M \\
g\left(t_{N}\right)
\end{array}\right], T=\left[\begin{array}{c}
T\left(t_{0}\right) \\
T\left(t_{1}\right) \\
M \\
T\left(t_{N}\right)
\end{array}\right]=\left[\begin{array}{cccc}
1 & t_{0} & L & t_{0}^{N} \\
1 & t_{1} & L & t_{1}^{N} \\
M & M & 0 & M \\
1 & t_{N} & L & t_{N}^{N}
\end{array}\right] .
$$

So, we can write the fundamental matrix equation (11) corresponding to Eq.(1) in the form

$$
\mathbf{W A}=\mathbf{G} \text { or }[\mathbf{W} ; \mathbf{G}] ; \mathbf{W}=\mathbf{T} \mathbf{C}^{T} \mathbf{R}^{T}-\lambda \mathbf{T} \mathbf{R}^{T}-\sum_{j=1}^{J} \mathbf{M}_{j} \mathbf{T B}\left(q_{j}\right) \mathbf{R}^{T}
$$

Here, Eq. (12) corresponds to a system of $N+1$ linear algebraic equations with $N+1$ unknown Morgan-Voyce coefficients $a_{0}, a_{1}, \ldots, a_{N}$. For the conditions (2), we can obtain the matrix form as,

$$
y(0)=\mathbf{T}(0) \mathbf{R}^{\mathbf{T}} \mathbf{A}=[\gamma]
$$

On the other hand, the matrix form for the condition can be written as

$$
\mathbf{U A}=[\gamma] \text { or }[\mathbf{U} ; \boldsymbol{\gamma}]
$$

where

$$
\mathbf{U}=\mathbf{T}(0) \mathbf{R}^{T}=\left[\begin{array}{lllll}
u_{0} & u_{1} & u_{2} & K & u_{N}
\end{array}\right] .
$$

Under the condition (2), to obtain the solution of Eq. (1), we replace the row matrice (13) by the last one row of the matrix (12), so have the new augmented matrix $[15,16,17]$

$$
[\widetilde{\mathbf{W}} ; \widetilde{\mathbf{G}}]=\left[\begin{array}{ccccccc}
w_{00} & w_{01} & w_{02} & K & w_{0 N} & ; & g\left(t_{0}\right) \\
w_{10} & w_{11} & w_{12} & K & w_{1 N} & ; & g\left(t_{1}\right) \\
w_{20} & w_{21} & w_{22} & K & w_{2 N} & ; & g\left(t_{2}\right) \\
M & M & M & M & M & M & M \\
w_{N-10} & w_{N-11} & w_{N-12} & K & w_{N-1 N} & ; & g\left(t_{N-1}\right) \\
u_{0} & u_{1} & u_{2} & K & u_{N} & ; & g\left(t_{N}\right)
\end{array}\right]
$$

If $\operatorname{rank} \widetilde{\mathbf{W}}=\operatorname{rank}[\widetilde{\mathbf{W}} ; \widetilde{\mathbf{G}}]=N+1$, then we can write $\mathbf{A}=(\widetilde{\mathbf{W}})^{-1} \widetilde{\mathbf{G}}$. Thus, we uniquely determine the matrix $\mathbf{A}$ (thereby the coefficients $\left.a_{0}, a_{1}, \ldots, a_{N}\right)$. So Eq. (1) with condition (2) has a unique solution and this solution is given by Morgan-Voyce series soluion (4). On the other hand, when $|\widetilde{\mathbf{W}}|=0$, that is if $\operatorname{rank} \widetilde{\mathbf{W}}=\operatorname{rank}[\widetilde{\mathbf{W}} ; \widetilde{\mathbf{G}}]<N+1$, then one can be found a particular solution. Otherwise if $\operatorname{rank} \widetilde{\mathbf{W}} \neq \operatorname{rank}[\widetilde{\mathbf{W}} ; \widetilde{\mathbf{G}}]<N+1$, then there is no solution.

\section{Residual correction and error estimation}

In this section, we will give an error estimation for the Morgan-Voyce polynomial solution (4) with the residual error function [23-26] and will improve the Morgan-Voyce polynomial solution (4) with the help of the residual error function. For this purpose, we get the residual function of the Morgan-Voyce collocation method as

$$
R_{N}(t)=L\left[y_{N}(t)\right]-g(t)
$$


Here $y_{N}(t)$ is the Morgan-Voyce polynomial solution given by (4) of problem (1) and (2). Thus, $y_{N}(t)$ satisfies the problem

$$
\begin{aligned}
L\left[y_{N}(t)\right] & =y_{N}^{\prime}(t)-\lambda y_{N}(t)-\sum_{j=1}^{J} \mu_{j}(t) y_{N}\left(q_{j} t\right) \\
& =g(t)+R_{N}(t) \\
y_{N}(0) & =\gamma .
\end{aligned}
$$

Also, the error function $e_{N}(t)$

$$
e_{N}(t)=y(t)-y_{N}(t)
$$

such that $y(t)$ is the exact solution of problem (1) and (2). By using Eqs. (1), (2), (15) and (16) we can get the error differential equation

$$
L\left[e_{N}(t)\right]=L[y(t)]-L\left[y_{N}(t)\right]=-R_{N}(t)
$$

with the condition

$$
e_{N}(0)=0
$$

or clearly, the error problem is

$$
\begin{array}{r}
e_{N}^{\prime}(t)-\lambda e_{N}(t)-\sum_{j=1}^{J} \mu_{j}(t) e_{N}\left(q_{j} t\right)=-R_{N}(t) \\
e_{N}(0)=0 .
\end{array}
$$

Here, we note that the nonhomogeneous condition

$$
y(0)=\gamma
$$

and

$$
y_{N}(0)=\gamma
$$

has been reduced to the homogeneous condition

$$
e_{N}(0)=0 \text {. }
$$

By solving problem (17)-(18) with the method introduced section (2) and (3), we get the approximation

$$
e_{N, M}(t)=\sum_{n=0}^{M} a_{n}^{*} B_{n}(t), \quad M \geq N
$$

to $e_{N}(t)$.

Consequently, by means of the polynomials $y_{N}(t)$ and $e_{N, M}(t),(M \geq N)$, we get the correct Morgan-Voyce polynomial solution $y_{N, M}(t)=y_{N}(t)+e_{N, M}(t)$. Also, we construct the error function $e_{N}(t)=y(t)-y_{N}(t)$, the correct error function $E_{N, M}(t)=e_{N}(t)-e_{N, M}(t)=y(t)-y_{N, M}(t)$ and the estimated error function $e_{N, M}(t)$.

If the exact solution of Eq. (1) is unknown, then the absolute errors $\left|e_{N}\left(t_{i}\right)\right|=\left|y\left(t_{i}\right)-y_{N}\left(t_{i}\right)\right|,\left(0 \leq t_{i} \leq b\right)$ are not found. However the absolute errors can be approximately computed with the aid of the estimated absolute error function $\left|e_{N, M}(t)\right|$. 


\section{Numerical examples}

In this section, to show the accuracy and effeciency properties of the present method we give several numerical examples. We have performed all the calculations with MATLAB. The values of the exact solution $y(t)$, the polynomial approximate solution $y_{N}(t)$, the corrected Morgan-Voyce polynomial solution $y_{N, M}(t)$, the absolute error function $e_{N}(t)=\left|y(t)-y_{N}(t)\right|$, the corrected absolute error function $\left|E_{N, M}(t)\right|$ and the estimated absolute error function $e_{N, M}(t)$ have been illustrated in Tables and Figures at the selected points of the given interval.

Example 1.With exact solution $y(t)=e^{t}$, consider the pantograph equation of first order

$$
y^{\prime}(t)=y(t)+\cos \left(\frac{t}{3}\right) y(0.2 t)-\sin \left(\frac{t}{5}\right) y(0.5 t)+\sin \left(\frac{t}{5}\right) e^{0.5 t}-\cos \left(\frac{t}{3}\right) e^{0.2 t}, \quad y(0)=1, \quad 0 \leq t \leq 1 .
$$

And approximate the solution by the truncated Morgan-Voyce series

$$
y_{3}(t)=\sum_{n=0}^{3} a_{n} B_{n}(t)
$$

where $N=3, \mu_{1}(t)=\cos \left(\frac{t}{3}\right), \mu_{2}(t)=-\sin \left(\frac{t}{5}\right), g(t)=\sin \left(\frac{t}{5}\right) e^{0.5 t}-\cos \left(\frac{t}{3}\right) e^{0.2 t}, q_{1}=0.2, q_{2}=0.5, \lambda=1$. From Section 3 , for $N=3$, the set of collocation points is,

$$
\left\{x_{0}=0, x_{1}=\frac{1}{3}, x_{2}=\frac{2}{3}, x_{3}=1\right\}
$$

and from Eq. (10), the fundamental matrix equation of the problem is

$$
\left\{\mathbf{T C}^{T} \mathbf{R}^{T}-\lambda \mathbf{T} \mathbf{R}^{T}-\mathbf{M}_{1} \mathbf{T B}\left(q_{1}\right) \mathbf{R}^{T}-\mathbf{M}_{2} \mathbf{T B}\left(q_{2}\right) \mathbf{R}^{T}\right\} \mathbf{A}=\mathbf{G}
$$

where

$$
\begin{aligned}
& \mathbf{M}_{1}=\left[\begin{array}{cccc}
1 & 0 & 0 & 0 \\
0 & \frac{967}{973} & 0 & 0 \\
0 & 0 & \frac{119}{122} & 0 \\
0 & 0 & 0 & \frac{3073}{3252}
\end{array}\right], \mathbf{M}_{2}=\left[\begin{array}{ccccc}
0 & 0 & 0 & 0 \\
0 & -\frac{90}{1351} & 0 & 0 \\
0 & 0 & -\frac{247}{1858} & 0 \\
0 & 0 & 0 & -\frac{209}{1052}
\end{array}\right], \mathbf{C}^{T}=\left[\begin{array}{llll}
0 & 1 & 0 & 0 \\
0 & 0 & 2 & 0 \\
0 & 0 & 0 & 3 \\
0 & 0 & 0 & 0
\end{array}\right], \mathbf{R}^{T}=\left[\begin{array}{llll}
1 & 0 & 0 & 0 \\
2 & 1 & 0 & 0 \\
3 & 4 & 1 & 0 \\
4 & 10 & 6 & 1
\end{array}\right], \mathbf{T}=\left[\begin{array}{cccc}
1 & 0 & 0 & 0 \\
1 & \frac{1}{3} & \frac{1}{9} & \frac{1}{27} \\
1 & \frac{2}{3} & \frac{4}{9} & \frac{8}{27} \\
1 & 1 & 1 & 1
\end{array}\right] \\
& \mathbf{G}=\left[\begin{array}{c}
-1 \\
-\frac{1143}{1162} \\
-\frac{615}{662} \\
-\frac{1006}{1217}
\end{array}\right], \mathbf{B}\left(q_{1}\right)=\mathbf{B}(0.2)=\left[\begin{array}{cccc}
1 & 0 & 0 & 0 \\
0 & \frac{1}{5} & 0 & 0 \\
0 & 0 & \frac{1}{25} & 0 \\
0 & 0 & 0 & \frac{1}{125}
\end{array}\right], \mathbf{B}\left(q_{2}\right)=\mathbf{B}(0.5)=\left[\begin{array}{cccc}
1 & 0 & 0 & 0 \\
0 & \frac{1}{2} & 0 & 0 \\
0 & 0 & \frac{1}{4} & 0 \\
0 & 0 & 0 & \frac{1}{8}
\end{array}\right]
\end{aligned}
$$

For this fundamental matrix equation, the augmented matrix is,

$$
[\mathbf{W} ; \mathbf{G}]=\left[\begin{array}{cccccc}
-2 & -3 & -2 & 2 & ; & -1 \\
-\frac{609}{316} & -\frac{3778}{1165} & -\frac{24281}{8726} & \frac{1477}{731} ; & -\frac{1143}{1162} \\
-\frac{2117}{1149} & -\frac{1454}{423} & -\frac{1756}{481} & \frac{603}{412} ; & -\frac{615}{662} \\
-\frac{1411}{808} & -\frac{1895}{529} & -\frac{1339}{292} & \frac{623}{3013} & ; & -\frac{1006}{1217}
\end{array}\right]
$$

From Eq. (13), the matrix forms for initial condition is

$$
\mathbf{U A}=[\gamma] \text { or }[\mathbf{U} ; \gamma]
$$

or briefly

$$
[\mathbf{U} ; \gamma]=\left[\begin{array}{ccccc}
1 & 0 & 0 & 0 & ;
\end{array}\right]
$$


From system (14), we can write the new augmented matrix based on conditions as

$$
[\widetilde{\mathbf{W}} ; \widetilde{\mathbf{G}}]=\left[\begin{array}{cccccc}
-2 & -3 & -2 & 2 & ; & -1 \\
-\frac{609}{316} & -\frac{3778}{1165} & -\frac{24281}{8726} & \frac{1477}{731} & ; & -\frac{1143}{1162} \\
-\frac{2117}{1149} & -\frac{1454}{423} & -\frac{1756}{481} & \frac{603}{412} & ; & -\frac{615}{662} \\
1 & 0 & 0 & 0 & ; & 1
\end{array}\right]
$$

If we solve this system, we obtain Morgan-Voyce coefficients matrix as follows

$$
A=\left[-\frac{2321}{1193} \frac{3561}{1472}-\frac{2602}{2749} \frac{169}{714}\right]^{T} .
$$

So, for $N=3$, the approximate solution of the problem yields

$$
y_{3}(t)=1+t+0.4736418259 t^{2}+0.2366946411 t^{3} .
$$

Now, let us find the improved Morgan-Voyce polynomial solution for $M=5$. For this purpose, let us first consider the error problem

$$
\begin{aligned}
e_{3}^{\prime}(t)-e_{3}(t)-\cos \left(\frac{t}{3}\right) e_{3}(0.2 t)+\sin \left(\frac{t}{5}\right) e_{3}(0.5 t)-\sin \left(\frac{t}{5}\right) e^{0.5 t} \cos \left(\frac{t}{3}\right)+e^{0.2 t} & =-R_{3}(t), \quad 0 \leq t \leq 1 \\
e_{3}(0) & =0
\end{aligned}
$$

where the residual error function is

$$
R_{3}(t)=y_{3}^{\prime}(t)-y_{3}(t)-\cos \left(\frac{t}{3}\right) y_{3}(0.2 t)+\sin \left(\frac{t}{5}\right) y_{3}(0.5 t)-g(t)
$$

By solving the error problem (20)-(21) for $M=5$ with the method introduced in Section 2 and 3, the Morgan-Voyce error function approximation

$$
\begin{aligned}
e_{3,5}(t) & =-1.75207071074 e-16-(7.086402501176031 e-3) t+(4.468056167244545233 e-2) t^{2} \\
& -(8.284377115551890685 e-2) t^{3}+(3.560908045406971714 e-2) t^{4} \\
& +(1.371093245056628252 e-2) t^{5}
\end{aligned}
$$

We have the improved Morgan-Voyce polynomial solution

$$
\begin{aligned}
y_{3,5}(t) & =0.9999999999999998+1.0000008834778 t+(4.99554494109247083 e-1) t^{2} \\
& +(1.6938589008435243 e-1) t^{3}+(3.560908045406971714 e-2) t^{4} \\
& +(1.371093245056628252 e-2) t^{5}
\end{aligned}
$$

Table 1 shows some numerical values of the exact solution, the Morgan-Voyce polynomial solution and the improved Morgan-Voyce polynomial solutions. In Table 2, the actual absolute errors are compared with the absolute errors estimated by the presented method for $N=3,6$ and $M=5,8,10,12$ and also the absolute error functions are compared in Figure 1 and 2. We see from these comparisons that the estimated absolute errors are quite close to the actual absolute errors. Table 3 denotes the absolute errors of the improved Morgan-Voyce polynomial solutions for $N=3,6$ and $M=5,8,10,12$. The improved absolute error functions are given in Figure 3 and 4. It is seen from Tables 2 and 3 and Figure 1 that the errors decrease when $N$ and $M$ are increased. 
Table 1: Numerical results of the exact and the approximate solutions for $N=3,6$ and $M=5,8,10,12$ of Eq.(19).

\begin{tabular}{ccccc}
\hline \multicolumn{2}{c}{ Exact Solution } & Morgan-Voyce solution & \multicolumn{2}{c}{ Present Method } \\
\hline$t_{i}$ & $y\left(t_{i}\right)=e^{t_{i}}$ & $y_{3}\left(t_{i}\right)$ & $y_{3,5}\left(t_{i}\right)$ & $y_{3,8}\left(t_{i}\right)$ \\
\hline 0.0 & 1 & 1 & 0.999999999999999 & 0.999999999999999 \\
0.2 & 1.22140275816 & 1.22140275816 & 1.221398805607721 & 1.22140275849008 \\
0.4 & 1.49182469764 & 1.49182469764 & 1.491821761821928 & 1.49182469826052 \\
0.6 & 1.82211880039 & 1.82211880039 & 1.822108599158451 & 1.82211880127839 \\
0.8 & 2.22554092849 & 2.22554092849 & 2.225519436434760 & 2.22554092965348 \\
1.0 & 2.71828182846 & 2.71828182846 & 2.718261280576066 & 2.71828183008548 \\
& & & & \\
\hline \hline & $y\left(t_{i}\right)=e^{t_{i}}$ & $y_{6}\left(t_{i}\right)$ & & $y_{6,12}\left(t_{i}\right)$ \\
& & & & \\
\hline 0 & 1 & 1 & 1.000000000000001 & 0.99999999999999 \\
0.2 & 1.22140275816 & 1.22140246213 & 1.221402758160364 & 1.22140275816017 \\
0.4 & 1.49182469764 & 1.49182428065 & 1.491824697641544 & 1.49182469764127 \\
0.6 & 1.82211880039 & 1.82211824351 & 1.822211880039085 & 1.82211880039051 \\
0.8 & 2.22554092849 & 2.22554021120 & 2.225540928492886 & 2.22554092849247 \\
1.0 & 2.71828182846 & 2.71828001904 & 2.718281828459822 & 2.71828182845905 \\
\hline
\end{tabular}

Table 2: Comparison of the absolute error functions for $\mathrm{N}=3,6$ and $\mathrm{M}=5,8,10,12$ of Eq. (19).

\begin{tabular}{|c|c|c|c|}
\hline \multicolumn{2}{|c|}{ Absolute errors for Morgan-Voyce solutions } & \multicolumn{2}{|c|}{ Estimated absolute errors for Morgan-Voyce solutions } \\
\hline$t_{i}$ & $\left|e_{3}\left(t_{i}\right)\right|=\left|y\left(t_{i}\right)-y_{3}\left(t_{i}\right)\right|$ & $e_{3,5}\left(t_{i}\right)$ & $\left|e_{3,8}\left(t_{i}\right)\right|$ \\
\hline 0.0 & 0 & $1.7521 \mathrm{e}-016$ & $1.5700 \mathrm{e}-016$ \\
\hline 0.2 & $2.2749 \mathrm{e}-004$ & $2.3145 \mathrm{e}-004$ & $2.2749 \mathrm{e}-004$ \\
\hline 0.4 & $6.7256 \mathrm{e}-005$ & $6.4320 \mathrm{e}-005$ & $6.7256 \mathrm{e}-005$ \\
\hline 0.6 & $3.6979 \mathrm{e}-004$ & $3.7999 \mathrm{e}-004$ & $3.6979 \mathrm{e}-004$ \\
\hline 0.8 & $3.8980 \mathrm{e}-004$ & $4.1130 \mathrm{e}-004$ & $3.8980 \mathrm{e}-004$ \\
\hline 1.0 & $4.0909 \mathrm{e}-003$ & $4.0704 \mathrm{e}-003$ & $4.0910 \mathrm{e}-003$ \\
\hline & $\left|e_{6}\left(t_{i}\right)\right|=\left|y\left(t_{i}\right)-y_{6}\left(t_{i}\right)\right|$ & $\left|e_{6,10}\left(t_{i}\right)\right|$ & $\left|e_{6,12}\left(t_{i}\right)\right|$ \\
\hline 0.0 & 0 & $4.7722 \mathrm{e}-016$ & $2.3261 \mathrm{e}-016$ \\
\hline 0.2 & $2.9603 \mathrm{e}-007$ & $2.9603 \mathrm{e}-007$ & $2.9603 \mathrm{e}-007$ \\
\hline 0.4 & $4.1700 \mathrm{e}-007$ & $4.1700 \mathrm{e}-007$ & $4.1700 \mathrm{e}-007$ \\
\hline 0.6 & $5.5688 \mathrm{e}-007$ & $5.5688 \mathrm{e}-007$ & $5.5688 \mathrm{e}-007$ \\
\hline 0.8 & $7.1730 \mathrm{e}-007$ & $7.1730 \mathrm{e}-007$ & $7.1730 \mathrm{e}-007$ \\
\hline 1.0 & $1.8094 \mathrm{e}-006$ & $1.8094 \mathrm{e}-006$ & $1.8094 \mathrm{e}-006$ \\
\hline
\end{tabular}

Table 3: Numerical results of the corrected error functions for $\mathrm{N}=3,6$ and $\mathrm{M}=5,8,10,12 \mathrm{Eq}$. of (19).

\begin{tabular}{ccc|cc|c}
\hline \multicolumn{7}{c}{ Improved absolute errors } \\
\hline$t_{i}$ & $\left|E_{3,5}\left(t_{i}\right)\right|$ & $\left|E_{3,8}\left(t_{i}\right)\right|$ & $E_{6,10}\left(t_{i}\right) \mid$ & $\left|E_{6,12}\left(t_{i}\right)\right|$ \\
\hline 0.0 & $2.2204 \mathrm{e}-016$ & $1.1102 \mathrm{e}-016$ & $4.4409 \mathrm{e}-016$ & $2.2204 \mathrm{e}-016$ \\
0.2 & $3.9526 \mathrm{e}-006$ & $3.2991 \mathrm{e}-010$ & $1.9451 \mathrm{e}-013$ & $2.2204 \mathrm{e}-016$ \\
0.4 & $2.9358 \mathrm{e}-006$ & $6.1925 \mathrm{e}-010$ & $2.7378 \mathrm{e}-013$ & $6.6613 \mathrm{e}-016$ \\
0.6 & $1.0201 \mathrm{e}-005$ & $8.8788 \mathrm{e}-010$ & $3.4117 \mathrm{e}-013$ & $8.8818 \mathrm{e}-016$ \\
0.8 & $2.1492 \mathrm{e}-005$ & $1.1610 \mathrm{e}-009$ & $4.1833 \mathrm{e}-013$ & $1.1102 \mathrm{e}-015$ \\
1.0 & $0.0548 \mathrm{e}-005$ & $1.6264 \mathrm{e}-009$ & $7.7638 \mathrm{e}-013$ & $4.4409 \mathrm{e}-016$ \\
\hline
\end{tabular}




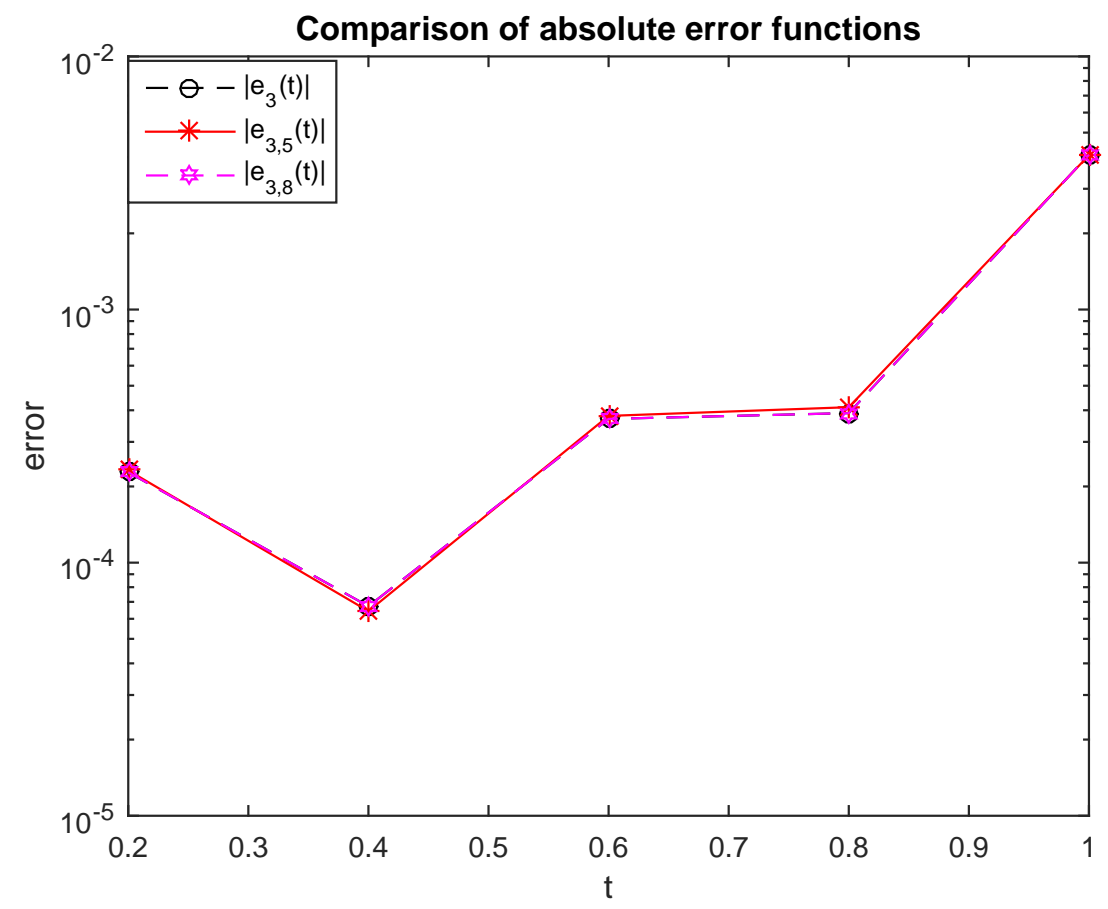

Fig. 1: Comparison of the absolute error functions and the estimated error functions for $N=6$ and $M=10,12$ of Eq. (19).

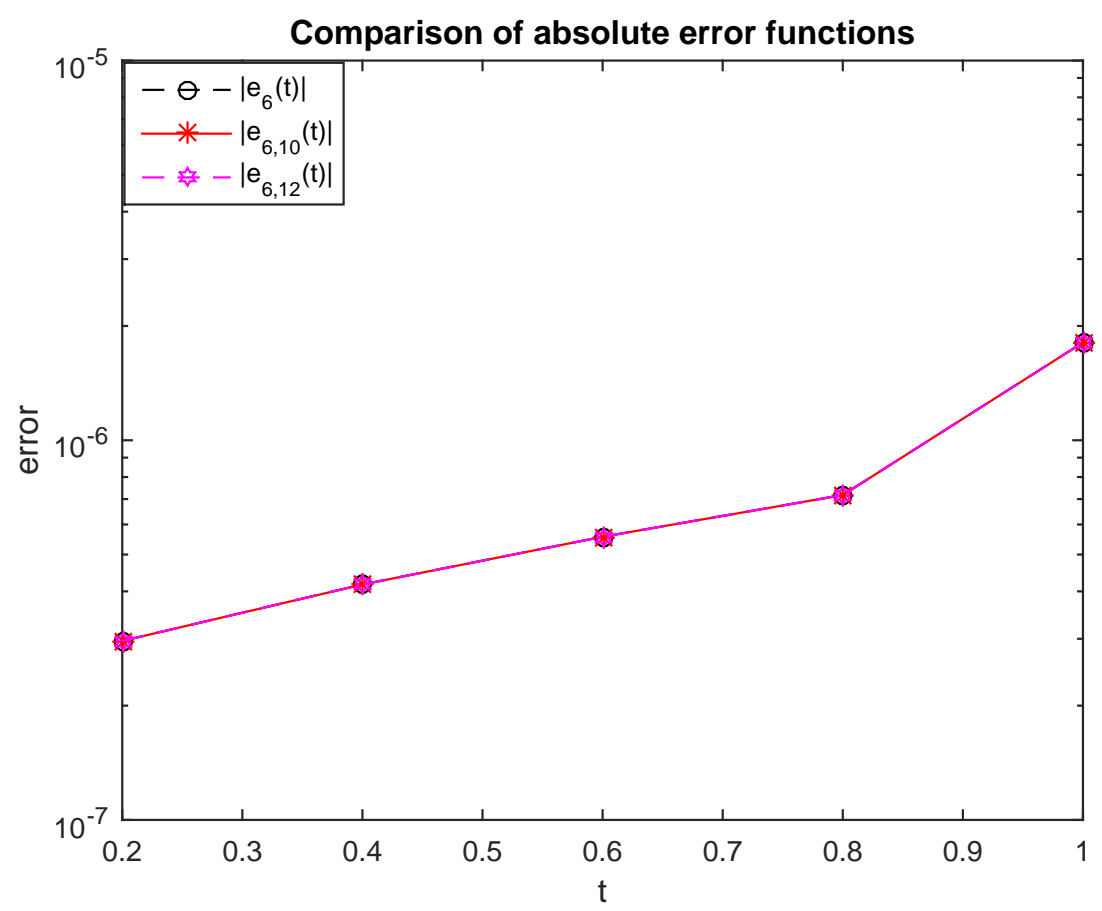

Fig. 2: Comparison of the absolute error functions and the estimated error functions for $N=6$ and $M=10,12$ of Eq. (19). 


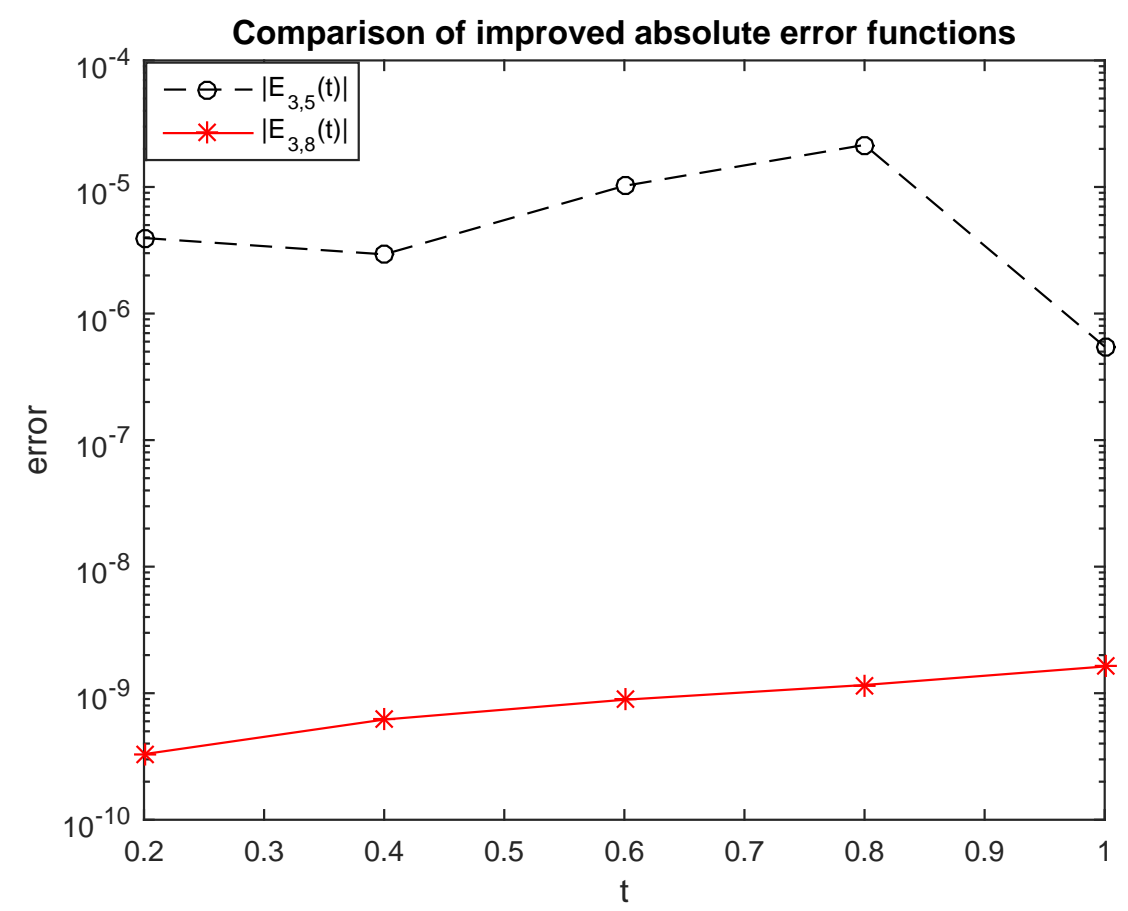

Fig. 3: Comparison of the improved absolute error functions for $N=3$ and $M=5,8$ of Eq. (19).

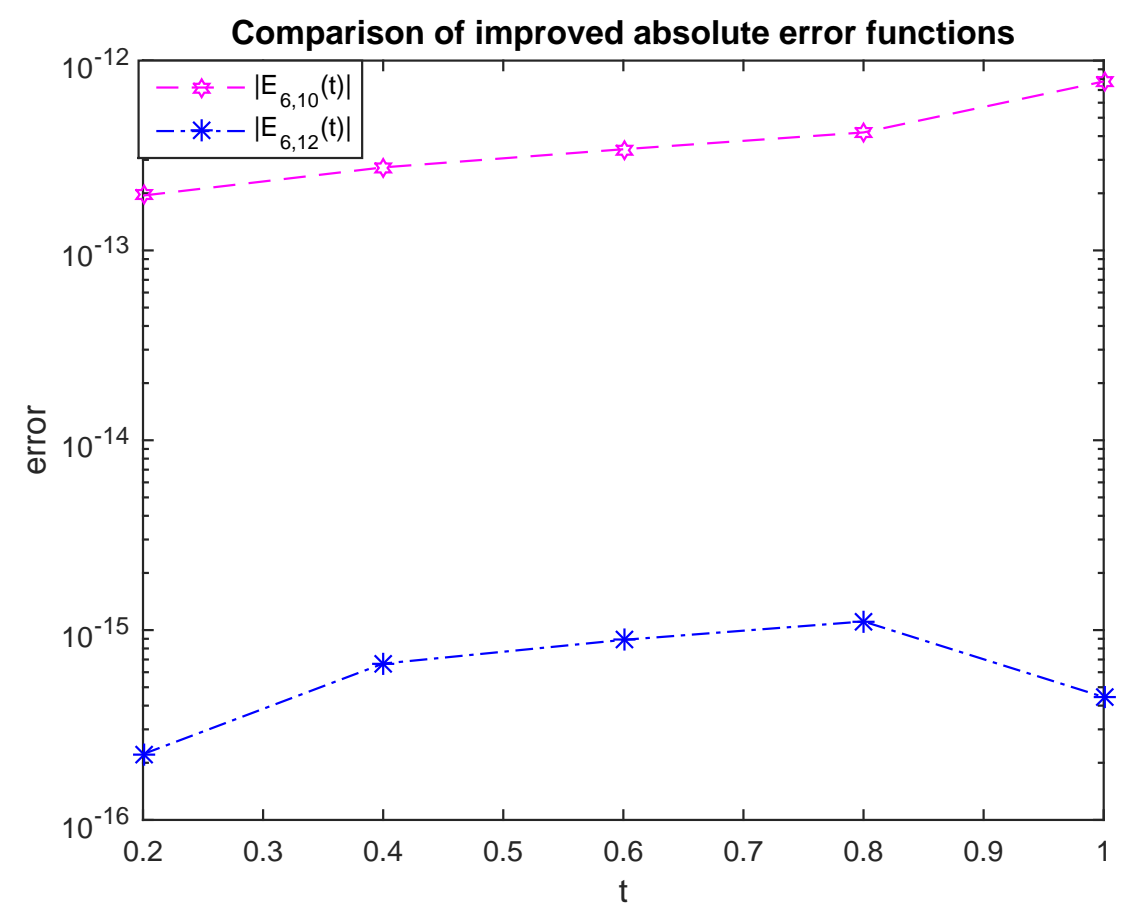

Fig. 4: Comparison of the improved absolute error functions for $N=6$ and $M=10,12$ of Eq. (19). 


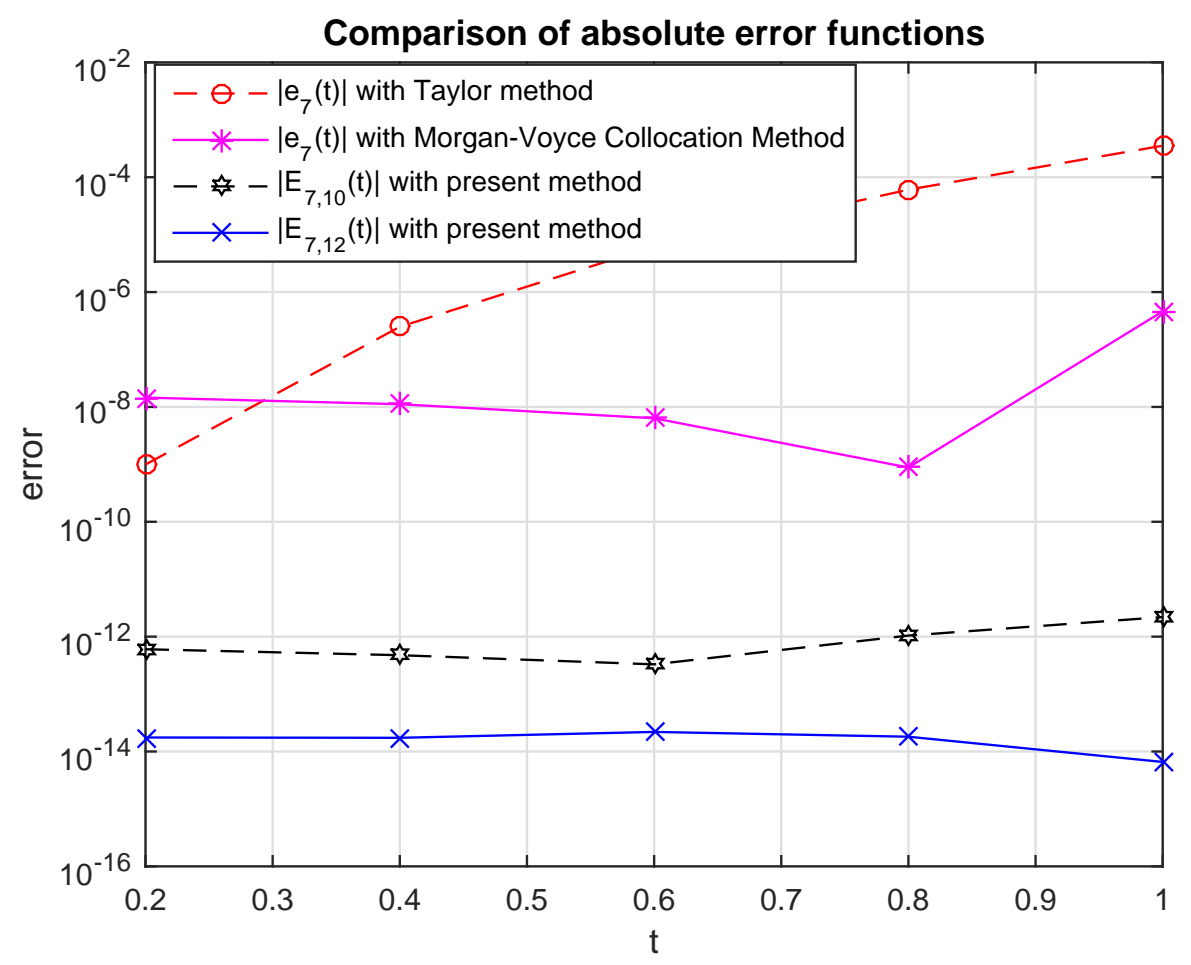

Fig. 5: Comparison of the absolute error functions $\left|e_{N}(t)\right|$ and $\left|E_{N, M}(t)\right|$ for $N=7$ and $M=10,12$ of Eq. (22).

Example 2. With the exact solution $y(t)=e^{-t} \cos (t)$, consider the pantograph equation with variable coefficients [19]

$$
y^{\prime}(t)=-y(t)+\mu_{1}(t) y\left(\frac{t}{2}\right)+\mu_{2}(t) y\left(\frac{t}{4}\right), \quad y(0)=1,0 \leq t \leq 1
$$

where $\mu_{1}(t)=-e^{-0.5 t} \sin (0.5 t), \mu_{2}(t)=-2 e^{-0.75 t} \cos (0.5 t) \sin (0.25 t)$. From Eq. (13), the fundamental matrix equation of the problem is

$$
\left\{\mathbf{T C}^{T} \mathbf{R}^{T}-\lambda \mathbf{T} \mathbf{R}^{T}-\mathbf{M}_{1} \mathbf{T B}\left(q_{1}\right) \mathbf{R}^{T}-\mathbf{M}_{2} \mathbf{T B}\left(q_{2}\right) \mathbf{R}^{T}\right\} \mathbf{A}=\mathbf{G}
$$

here $\mu_{1}(t)=-e^{-0.5 t} \sin (0.5 t), q_{1}=\frac{1}{2}, \mu_{2}(t)=-2 e^{-0.75 t} \cos (0.5 t) \sin (0.25 t), q_{2}=\frac{1}{4}, \lambda=-1, g(t)=0$.

According to the procedure in Section 2,3 and 4, we find the aproximate solutions of the problem for different values of $N$ and $M$. In Table 4, we compare the absolute errors obtained by the present method, the Morgan-Voyce collocation method and Taylor method. The absolute error functions are compared in Figure 5.

Table 4: Comparison of the solutions and the absolute errors of Eq. (22).

\begin{tabular}{ccccc}
\hline \multicolumn{2}{c}{ Taylor method } & Morgan-Voyce Collocation Method & \multicolumn{2}{c}{ Present method } \\
\hline$t_{i}$ & $N=7,\left|e_{7}\left(t_{i}\right)\right|$ & $N=7,\left|e_{7}\left(t_{i}\right)\right|$ & $\left|E_{7,10}\left(t_{i}\right)\right|$ & $\left|E_{7,12}\left(t_{i}\right)\right|$ \\
\hline 0.0 & 0 & 0 & $4.3110 \mathrm{e}-14$ & $1.2724 \mathrm{e}-14$ \\
0.2 & $9.9331 \mathrm{e}-010$ & $1.4522 \mathrm{e}-008$ & $6.0341 \mathrm{e}-13$ & $1.7542 \mathrm{e}-14$ \\
0.4 & $2.4854 \mathrm{e}-007$ & $1.1150 \mathrm{e}-008$ & $4.7518 \mathrm{e}-13$ & $1.7319 \mathrm{e}-14$ \\
0.6 & $6.2234 \mathrm{e}-006$ & $6.3543 \mathrm{e}-009$ & $3.2929 \mathrm{e}-13$ & $2.1927 \mathrm{e}-14$ \\
0.8 & $6.0719 \mathrm{e}-005$ & $8.8224 \mathrm{e}-010$ & $1.0442 \mathrm{e}-12$ & $1.8097 \mathrm{e}-14$ \\
1.0 & $3.5341 \mathrm{e}-004$ & $4.6167 \mathrm{e}-007$ & $2.1931 \mathrm{e}-12$ & $6.5781 \mathrm{e}-15$ \\
\hline
\end{tabular}




\section{Conclusion}

In this article, we have improved the Morgan-Voyce collocation method, based on Morgan-Voyce polynomials, for Multi-pantograph equations. This improvement is based on the residual error function. In addition, an error estimation is given with the residual error function. Morever, if the exact solution of the problem is unknown, then the absolute errors $\left|e_{N}\left(t_{i}\right)\right|=\left|y\left(t_{i}\right)-y_{N}\left(t_{i}\right)\right|,\left(0 \leq t_{i} \leq b\right)$ can be estimated by the approximation $\left|e_{N, M}(t)\right|$. It is seen from Tables 1-3 that the estimated absolute errors $\left|e_{N, M}\left(t_{i}\right)\right|$ are quite close to the actual absolute errors $\left|e_{N}\left(t_{i}\right)\right|=\left|y\left(t_{i}\right)-y_{N}\left(t_{i}\right)\right|$. We see from tables and figures that the errors decrease when $N$ and $M$ are increased. The comparisons of the present method by the other methods show that our method is very effective. A consirable advantage of the method is that the approximate solutions are computed very easily by using a well-known symbolic software such as Matlab, Maple and Mathematica.

\section{Competing interests}

The authors declare that they have no competing interests.

\section{Authors' contributions}

All authors have contributed to all parts of the article. All authors read and approved the final manuscript.

\section{References}

[1] J.R. Ockendon and A.B. Tayler, The Dynamics of a current collection system for an electric locomotive, Proc R Soc London Ser A 322 (1971), 447-468.

[2] G.A. Derfel and F. Vogl, On the asymptotics of solutions of a class of linear functional-differential equations, Eur J Appl Maths 7 (1996),511-518.

[3] Feldstein and Y. Liu, On neutral functional-differenrial equations with variable time delays, Math Proc Cambridge Philos Soc 124 (1998), 371-384

[4] G.R. Morris, A. Feldstein, and E. W. Bowen, The Phragmen-Lindel' of principle and a class of functional-differential equations, Proceedings of NRL-MRC Conference on Ordinary Differential Equations, Academic Press, New York, 1972,pp. 513-540.

[5] G. Derfel and A. Iserles, The pantograph equaiton in the complex plane, J Math Anal Appl 213 (1997),117-132.

[6] W. G. Ajello, H. I. Freedman, and J. Wu, A model of stage structured population growth with density depended time delay, SIAM J Appl Math 52 (1992), 855-869.

[7] M. D. Buhmann and A. Iserles, Stability of the discretized pantograph differential equation, Math Comput 60 (1993), 575-589.

[8] L. Fox, D. F. Mayers. J. A. Ockendon, and A. B. Tayler, On a functional differential equation, J Inst Math Appl 8 (1971), $271-307$.

[9] G. Derfel, On compactly supported solutions of a class of functional-differential equations, Modern problems of functions theory and functional analysis, Karaganda University Press, 1980, (in Russian).

[10] G. Derfel, N. Dyn, and D. Levin, Generalized refinement equation and subdivision process, J Approx Theory 80 (1995), $272-297$.

[11] M. Sezer, Taylor polynomial solution of Volterra integral equations, Int. J. Math. Educ. Sci. Technol. 25 (1994) 625-633

[12] B. Bülbül, M. Gülsu, M. Sezer, A New Taylor collocation method for nonlinear Fredholm-Volterra integro-differential equations, Numer. Methods for partial Diff. Eq. (2009) doi: 10.1002/num.20470.

[13] A. Karamete, M. Sezer, A Taylor collocation method for the solution of linear integro-differential equations, Int. J. Comput. Math. 79 (9) (2002) 987-1000.

[14] M. Sezer, M. Gülsu, Polynomial solution of the most general linear Fredholm integro-differential-difference equation by means of Taylor matrix method. Int. J. Complex Var. 50 (5) (2005) 367-382

[15] N. Kurt and M. Sezer, Polynomial solution of high-order linear Fredholm integro-differential equations with constant coefficients, J Franklin Inst 345 (2008),839-850.

[16] M. Sezer and A. Akyüz-Daşcıoğlu, A Taylor method for numerical solution of generalized pantograph equations with linear functional argument, J Comput Appl Math 200 (2007), 217-225 
[17] M. Gülsu, Y. Öztürk, M. Sezer, A New collocation method for solution of mixed linear itegro-differential-difference equations, Appl. Math. Comput.(2010), in press, doi:10.1016/j.amc.2010.03.054.

[18] Ş. Yüzbaşı, Bessel polynomial solutions of linear differential, integral and integro- differential equations, MSc Thesis, Graduate School of Natural and Applied Sciences, Muğla Uni.,2009.

[19] M. Z. Liu and D. Li, Properties of analytic solution and numerical solution and multi-pantograph equation, Appl Math Comput 155 (2004), 853-871.

[20] D. J. Evans and K. R. Raslan, The Adomain decomposition method for solving delay differential equation, Int J Comput Math 82 (2005), 49-54.

[21] Y. Muroya, E. Ishiwata, and H. Brunner, On the attainable order of collocation methods for pantograph integro-differential equations, J Comput Appl Math 152 (2003),347-366.

[22] A. Akyüz-Daşçığlu and H. Ç. Yaslan, An approximation method for solution of nonlinear integral equations, Appl. Math. Comput. 174 (2006) 619-629.

[23] F.A. Oliveira, Collocation and residual correction, Numer. Math. 36 (1980) 27-31.

[24] İ. Çelik, Approximate calculation of eigenvalues with the method of weighted residuals-colllocation method, Appl. Math. Comput. 160 (2005) 401-410.

[25] S. Shahmorad, Numerical solution of the general form linear Fredholm-Volterra integro-differential equations by the Tau method with an error estimation, Appl. Math. Comput. 167 (2005) 1418-1429

[26] İ. Çelik, Collocation method and residual correction using Chebyshev series, Appl. Math. Comput. 174 (2006) 910-920. 